\title{
Patient-provider concordance in the perception of illness and disease: a cross-sectional study among multimorbid patients and their general practitioners in Switzerland
}

\author{
This article was published in the following Dove Press journal: \\ Patient Preference and Adherence \\ 22 August 2017 \\ Number of times this article has been viewed
}

\section{Stefan Neuner-Jehle \\ Stefan Zechmann \\ Daniela Grundmann \\ Maissen \\ Thomas Rosemann \\ Oliver Senn}

Institute of Primary Care, University of Zurich, Zurich, Switzerland
Correspondence: Stefan Neuner-Jehle Institute of Primary Care, University of Zurich, Pestalozzistrasse 24, 809। Zurich, Switzerland

$\mathrm{Tel}+4 \mid 442559855$

Fax +4I 442559097

Email stefan.neuner-jehle@usz.ch
Background: Multiple chronic health conditions are leading to multiple treatment procedures and polypharmacy. Prioritizing treatment according to patients' needs and preferences may be helpful for deprescribing. Thus, for improving health care, it is crucial for general practitioners (GPs) to perceive the chief complaints (CCs) of patients. The primary aim of this study was to investigate the patient-provider concordance of CCs and the secondary aim was to investigate the concordance between CCs and diagnosis, in a sample of Swiss multimorbid patients.

Materials and methods: A cross-sectional analysis based on a cluster randomized controlled trial (RCT) among 46 GPs, recruited between March 2015 to July 2016, and 334 multimorbid patients ( $\geq 60$ years taking $\geq 5$ drugs for at least 6 months) in Northern Switzerland was performed. CCs listed by GPs and by patients $(n=128)$ were classified according to the International Classification of Primary Care, version 2 (ICPC-2) coding system on chapter and component level and defined as concordant if ICPC-2 codes of patients and GPs were identical. Concordance was classified into full, moderate or low, depending on the ranking of patients' CCs on GPs' list. As secondary outcome, we compared patients' CCs to GPs' diagnosis. Statistics included descriptive measures and a multivariate regression analysis of factors that are modifying concordance.

Results: The mean age of patients was 76.9 (SD 8.1) years, where $38 \%$ were male, taking 7.9 (SD 2.6) drugs on the long term. The most frequent complaint was pain. Concordance of the CC was given in 101/128 (78.9\%) on the ICPC-2 chapter level, whereby $86 / 128(67.2 \%)$ showed full, 8/128 (6.3\%) moderate and 7/128 (5.5\%) low concordance; $27 / 128(21.1 \%)$ were discordant. Concordance between CCs and diagnosis was 53.6\%. Concordance increased with the intensity of the $\mathrm{CC}$ rated by patients (OR 1.48, CI 1.13-1.94, $P<0.001$ ). The younger age and higher intake of drugs were significantly associated with an increased concordance between CCs and diagnosis.

Conclusion: A majority of GPs perceive the CCs of the multimorbid patients correctly, but there is room for improvement.

Keywords: deprescribing, multimorbidity, patient-provider concordance, chief complaint

\section{Plain language summary}

Several long-term health conditions in a patient are leading to several treatment procedures, unless carefully aligned to patients' needs and preferences. Thus, for improving health care, it is crucial for general practitioners (GPs) to perceive the main complaints of patients. The aim of our study was to investigate how well Swiss GPs perceived the main complaints of their 
patients with several diseases. For this purpose, we compared 128 main complaints listed by patients to those listed by their GPs and investigated what factors influenced the degree of agreement or disagreement. The most frequent complaint was pain. Nearly $80 \%$ of patients and GPs agreed on the patient's main complaint; only one-fifth had different opinions on this matter. The more intensively patients perceived their main complaint, the higher was the agreement. Thus, a majority of GPs perceive the main complaint of the long-term patient correctly, but there is room for improvement. Increasing GPs' perception of these patients' suffering is crucial for improving their health care quality.

\section{Background}

Multimorbidity, defined as the co-occurrence of multiple chronic health conditions in a patient, is a challenge for health care systems, providers and patients. Multiple chronic health conditions are inevitably leading to multiple treatment procedures, for example, polypharmacy. ${ }^{1}$ Prioritizing chronic conditions and adapting treatment strategies according to patients' needs and preferences may be helpful for deprescribing. To deal with the complexity of these multiple health conditions within the sparse time of an encounter is an important and difficult task for general practitioners (GPs). ${ }^{2,3}$ Due to the complexity of these multiple conditions, GPs may underestimate or even miss the chief complaints (CCs) of patients. ${ }^{3}$

Considering patients' needs and preferences with regard to their $\mathrm{CC}$ is a central issue in models such as patient centeredness ${ }^{4}$ and patient-centered medical home. ${ }^{5}$ A high provider-patient concordance in the perception of disease and illness positively affects patient satisfaction, adherence to treatment and disease outcomes. ${ }^{5-7}$ Accordingly, researchers recently published a call in this journal for a better concordance between physicians and patients with regard to patients' needs and treatment goals. ${ }^{8}$ Thus, for improving health care, a high provider-patient concordance of CCs is important. There are only few research results available on the provider-patient concordance or discordance concerning complaints and/or diagnoses. ${ }^{9-11}$ GPs seem to focus more on the prognosis and treatment priorities than on the current health priorities of their elderly patients. ${ }^{11,12}$

The aim of our study was to investigate, in a sample of multimorbid Swiss patients, 1) to what degree GPs and patients were concordant in the perception of patients' CCs and 2) how concordant CCs were with the GPs' diagnoses ranked by relevance. Furthermore, we investigated the association between the concordance and patient/GP characteristics.

\section{Materials and methods Setting, design and participants}

For this cross-sectional study, we considered the first consultation dataset of a cluster randomized controlled study among GPs and their multimorbid patients in Northern Switzerland. ${ }^{13}$ GPs were recruited between March 2015 and July 2016. Patients were eligible if they were 60 years of age or older, listed in the GPs' practice card file and taking five or more drugs during, at least, the last 6 months (as a proxy for multimorbidity). Exclusion criteria were a life expectancy of less than 12 months or a substantial cognitive impairment. All participants provided written informed consent. For the purposes of the study, GPs were randomized into an intervention and a control group to measure the effects of an intervention to reduce drugs. Immediately after the first consultation with the patient, GPs and patients were asked by questionnaire a set of questions concerning drugs, diagnoses, $\mathrm{CC}$ of the patient, drugs changed during/after the encounter, quality of life, socioeconomic variables and time required for the consultation. ${ }^{13}$ Data were collected by the web-based data management program SecuTrial ${ }^{\circledR}$ (version 5.0.1, 2016; interActive Systems GmbH, Berlin, Germany). ${ }^{14}$ The study protocol was approved by the ethics committee of the Canton of Zurich (reference KEK-ZH-number 2014-0595).

\section{Outcomes and measurements}

The CCs were measured 1) in the GPs' questionnaires by a four-part list including ranking with regard to relevance, asking: "What are the CCs in the view of your patient?" (intervention group only, as this list was considered to be a part of the intervention), 2) in the patients' questionnaires by one question: "Which is your CC?" In addition, the severity of the $\mathrm{CC}$ was rated by the patient using a visual analog scale (VAS) ranging from 0 to 10 . The four most important medical diagnoses were measured in the GPs' questionnaires by asking for a four-part diagnosis list (ranked by importance for the patient's health; Figure 1).

The primary outcome was the concordance between patients and GPs concerning patients' CCs. The CCs were classified according to the International Classification of Primary Care, version 2 (ICPC-2) classification system ${ }^{15}$ on chapter and component level. Concordance was defined as the ICPC-2 code of patient's CC being identical with one of the CC codes on the four-part GP's list. We classified concordance into full, moderate or low, depending on the patient's CC code ranked first, second or third/fourth on the 


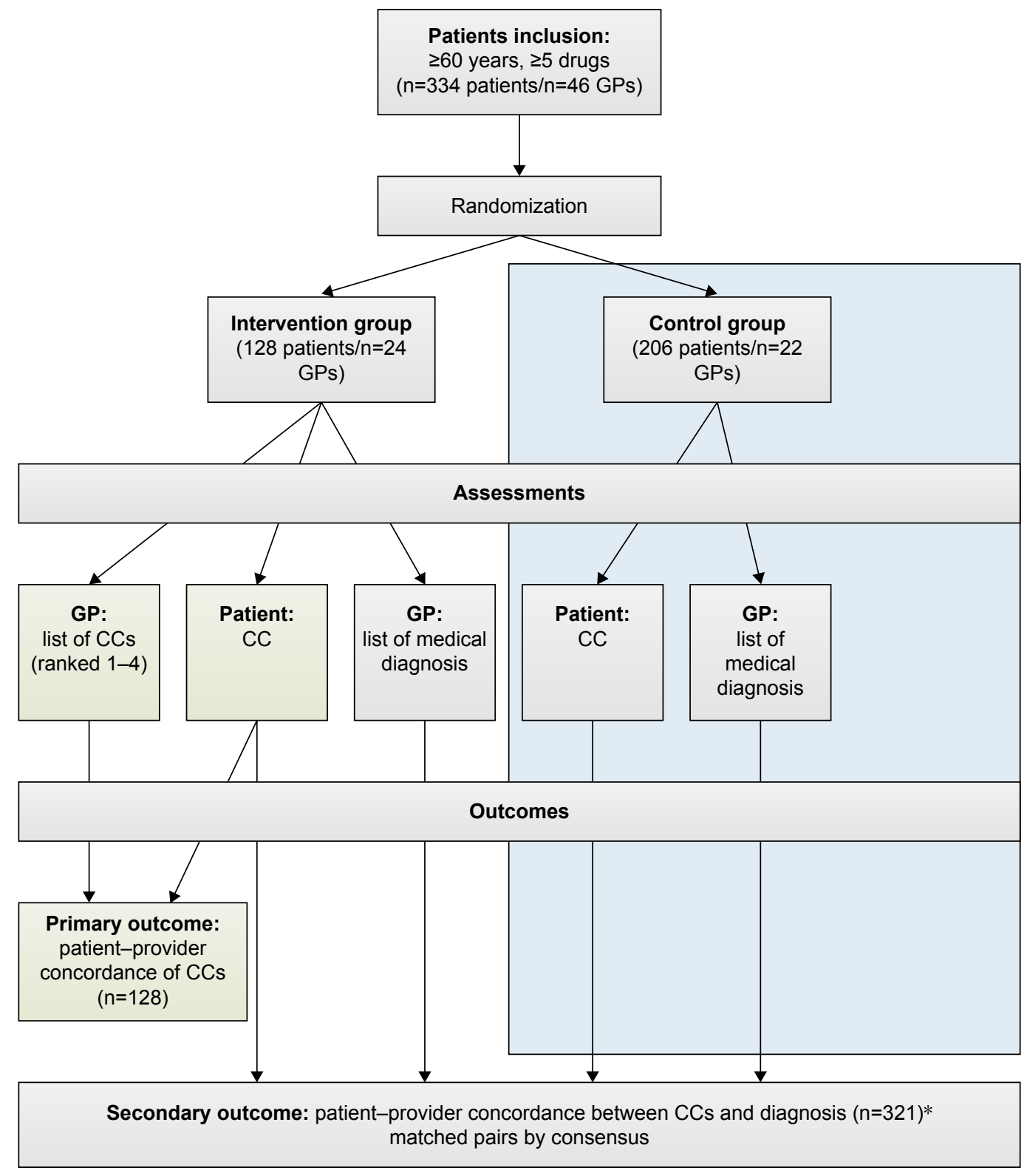

Figure I Study flowchart, assessments and outcomes.

Notes: *In two cases, data were missing; in II cases, patients declared no complaint at all.

Abbreviations: CC, chief complaint; GP, general practitioner.

GP's list. We defined discordance if the patient's CC code was not listed on GP's list at all.

The secondary outcome was the concordance between patients' CCs and GPs' diagnoses. Diagnoses were coded according to an adapted version of the classification system by van den Bussche et $\mathrm{al}^{16}$ developed for multimorbid elderly patients. We matched CCs to diagnosis based on clinical expertise (by consensus in our research team; Table 1). We defined concordance if there were matching pairs and discordance if not, according to the assignment. Concordance was classified into full, moderate or low, depending on the patient's CC code matching with the diagnoses on the GP's list ranked first, second or third/higher. Further covariates and measurements of patients and GPs are depicted in Table 2 .

\section{Statistical analysis}

For the descriptive analysis of GPs' and patients' characteristics, we used numbers and percentages for categorical variables and mean and SD for continuous variables. For group comparison of continuous variables, we used the Student's 
Table I Assignment of complaints to diagnosis, based on clinical expertise, by consensus

\begin{tabular}{ll}
\hline $\begin{array}{l}\text { Complaint on chapter } \\
\text { level (according to }\end{array}$ & Diagnosis on chapter level* \\
ICPC-2) & \\
\hline A: General and unspecific & Various \\
B: Blood, blood forming organs & Hematologic/oncological diseases \\
and immune mechanism & Venous diseases \\
D: Digestive & Digestive diseases \\
F: Eye & Neuropathies, psychopathies, \\
H: Ear & eye/ear diseases \\
K: Circulatory & CVDs \\
L: Musculoskeletal disorders & Rheumatological - bone diseases \\
N: Neurological & Neuropathies, psychopathies, \\
P: Psychological & eye/ear diseases \\
R: Respiratory & Respiratory diseases \\
S: Skin & Various \\
T: Endocrine/metabolic and & Metabolic diseases \\
nutritional & \\
U: Urological & Urological diseases \\
W: Pregnancy, childbearing, & Various \\
family planning & \\
X: Female genital & Sexual diseases \\
Y: Male genital & \\
Z: Social problems & Various \\
\hline
\end{tabular}

Notes: List of CCs classified on chapter level using the ICPC-2 coding system; ${ }^{15}$ *diagnosis coded on chapter level according to an adapted version of the classification system by van den Bussche et al. ${ }^{16}$

Abbreviations: CCs, chief complaints; CVDs, cardiovascular diseases; ICPC-2, International Classification of Primary Care, version 2.

$t$-test; for categorical variables, we used the Chi-square test (defining significance level as $P<0.05$ ). We performed a multivariate regression analysis to explore independent determinants of concordance. Since the full regression (all available variables) would result in a loss of power, we decided to perform a regression model with a predefined selection of eight variables of interest (Table 3 ) chosen with regard to clinical relevance and plausibility by research team consensus. In addition, we used a model based on a testing procedure (stepwise regression) to identify discrepancies to the model of interest. All calculations were performed with the statistical program $\mathrm{R}^{\circledR}$, version 3.2. ${ }^{17}$

\section{Results}

The mean age of the 334 patients was 76.9 (SD 8.1) years, where $38 \%$ were male, taking 7.9 (SD 2.6) drugs on the long term, and the mean age of the 46 GPs was 49.4 (SD 9.3) years, where $65 \%$ were male, working in practice since 13.6 (SD 9.7) years (Table 2). The most frequent CCs in patients' view on ICPC-2 component level $(n=321)$ were pain $(42.1 \%)$, weakness/tiredness $(6.3 \%)$, shortness of breath $(5.4 \%)$ and dizziness $(3.9 \%)$.
Table 2 Characteristics of patients and GPs included

\begin{tabular}{|c|c|}
\hline Patients & 334 \\
\hline \multicolumn{2}{|l|}{ Sex } \\
\hline Male, n (\%) & $152(46)$ \\
\hline Age (years), mean (SD) & $76.18(8.45)$ \\
\hline Weight $(\mathrm{kg})$, mean $(\mathrm{SD})$ & $76.80(16.06)$ \\
\hline \multicolumn{2}{|l|}{ Blood pressure (mmHg), mean (SD) } \\
\hline Systolic & $137.65(18.7)$ \\
\hline Diastolic & $75.44(12.08)$ \\
\hline Hemoglobin (g/dl), mean (SD)* & $12.84(1.56)$ \\
\hline $\mathrm{TSH}(\mathrm{mU} / \mathrm{L})$, mean $(\mathrm{SD})^{*}$ & $2.15(1.54)$ \\
\hline HbAlc (\%), mean (SD)* & $7.30(1.44)$ \\
\hline Number of drugs, mean (SD) & $8.46(2.94)$ \\
\hline Quality of life scale, mean (SD) $)^{\S}$ & $0.77(1.55)$ \\
\hline Quality of life (EQ-5D), mean (SD) ${ }^{\Phi}$ & $65.83(17.86)$ \\
\hline Severity of complaint VAS, mean (SD) ${ }^{\circ}$ & $4.18(2.50)$ \\
\hline \multicolumn{2}{|l|}{ Living situation, n (\%) } \\
\hline Living at home alone & $119(36)$ \\
\hline Living at home with family & $173(52)$ \\
\hline Living in nursing home & $38(11)$ \\
\hline GPs & 46 \\
\hline \multicolumn{2}{|l|}{ Sex } \\
\hline Male, n (\%) & $30(65)$ \\
\hline Age (years), mean (SD) & $49.42(9.32)$ \\
\hline Years of working experience, mean (SD) & $13.62(9.73)$ \\
\hline Working in single practice, $\mathrm{n}(\%)$ & $10(22)$ \\
\hline Practice connected to network, n (\%) & $21(46)$ \\
\hline
\end{tabular}

Notes: *Hemoglobin and TSH were documented only if patients received either iron/vitamin $\mathrm{Bl} 2$ or thyroid hormone substitution. HbAlc only in patients with known diabetes. ${ }^{85}$-point Likert scale $(-2$ to +2$) .{ }^{\oplus}(0-100) .{ }^{\circ}$ VAS (0-10).

Abbreviations: GP, general practitioner; TSH, thyroid-stimulating hormone; VAS, visual analog scale; EQ-5D, EuroQol-5 dimensions quality of life scale.

The most relevant diagnoses ( $\mathrm{n}=334)$ ranked first by GPs were cardiovascular diseases (CVDs; 40.1\%), metabolic diseases (17.7\%), rheumatological/bone diseases (14.7\%), neuropathies, psychopathies or eye/ear diseases $(9.6 \%)$ and hematological/oncological diseases (4.2\%; Figure 2).

CCs were concordant between patients and GPs in $101 / 128(78.9 \%)$ on the ICPC-2 chapter level. Of these, $86 / 128$ (67.2\%) showed full, 8/128 (6.3\%) moderate and $7 / 128$ (5.5\%) low concordance. On the ICPC-2 component level, concordance of CC was given in 83/128 (64.8\%). Of these, $72 / 128$ (56.3\%) showed full, 6/128 (4.7\%) moderate and 5/128 (3.9\%) low concordance. In 27/128 (21.1\%) pairs, there was discordance (Figure 3).

Concordance between patients' ratings of CCs and GPs' ranked diagnoses on chapter level was 174/321 (54.2\%). Of these, 60/321 (18.7\%) showed full, 36/321 (11.2\%) moderate and 76/321 (23.7\%) low concordance. In 147/321 (45.8\%) pairs, there was discordance.

On the ICPC-2 chapter level, concordance of CCs significantly increased with the intensity of the $\mathrm{CC}$ in patient's perception (OR 1.48, CI 1.13-1.94, $P<0.001$ ), 
Table 3 Multivariate regression

\begin{tabular}{|c|c|c|c|}
\hline Covariate & OR & $95 \% \mathrm{Cl}$ & $P$-value \\
\hline \multicolumn{4}{|c|}{ (A) Concordance of CCs on ICPC-2 chapter level $(n=I \mid 8)$} \\
\hline Patient's age & 1.03 & $0.97-1.10$ & 0.27 \\
\hline Patient's sex (male) & 0.54 & $0.19-1.53$ & 0.24 \\
\hline Number of drugs & 1.05 & $0.86-1.29$ & 0.64 \\
\hline Quality of life scale ${ }^{\S}$ & 1.40 & $0.74-2.64$ & 0.30 \\
\hline Quality of life (EQ-5D) $)^{\Phi}$ & 0.99 & $0.95-1.02$ & 0.54 \\
\hline Severity of complaint scale ${ }^{\circ}$ & 1.48 & $1.13-1.94$ & $<0.01$ \\
\hline Doctors' years in practice* & 0.92 & $0.83-1.03$ & 0.14 \\
\hline Doctors' age* & 1.02 & $0.91-1.15$ & 0.72 \\
\hline \multicolumn{4}{|c|}{ (B) Concordance of CCs on ICPC-2 component level ( $n=\mid$ I 8) } \\
\hline Patient's age & 1.04 & $0.99-1.10$ & 0.11 \\
\hline Patient's sex (male) & 0.73 & $0.31-1.72$ & 0.48 \\
\hline Number of drugs & 1.00 & $0.85-1.17$ & 0.96 \\
\hline Quality of life scale s $^{\S}$ & 1.27 & $0.76-2.13$ & 0.36 \\
\hline Quality of life (EQ-5D) $)^{\Phi}$ & 1.00 & $0.97-1.03$ & 0.86 \\
\hline Severity of complaint scale ${ }^{\circ}$ & 1.18 & $0.95-1.46$ & 0.13 \\
\hline Doctors' years in practice* & 0.92 & $0.84-1.02$ & 0.11 \\
\hline Doctors' age* & 1.05 & $0.94-1.17$ & 0.35 \\
\hline \multicolumn{4}{|c|}{ (C) Concordance between CCs and diagnosis ranked first } \\
\hline Patient's age & 0.96 & $0.94-0.99$ & 0.01 \\
\hline Patient's sex (male) & 0.90 & $0.56-1.44$ & 0.66 \\
\hline Number of drugs & 1.14 & $1.04-1.26$ & 0.01 \\
\hline Quality of life scale ${ }^{\S}$ & 0.86 & $0.66-1.13$ & 0.28 \\
\hline Quality of life (EQ-5D) $)^{\Phi}$ & 1.00 & $0.99-1.02$ & 0.61 \\
\hline Severity of complaint scale ${ }^{\circ}$ & 0.97 & $0.87-1.08$ & 0.58 \\
\hline Doctors' years in practice ${ }^{* *}$ & 1.00 & $0.95-1.06$ & 0.86 \\
\hline Doctors' age ${ }^{* *}$ & 1.01 & $0.95-1.07$ & 0.82 \\
\hline
\end{tabular}

Notes: Numbers $(\mathrm{n})$ refer to complete cases. ${ }_{\mathrm{n}} \mathrm{n}=20, * *_{\mathrm{n}}=40$. ${ }_{5}$-point Likert scale $(-2$ to +2$) .{ }^{\Phi}(0-100)$. ${ }^{\circ}$ VAS $(0-10)$. Data in bold indicates statistical significance. Abbreviations: CCs, chief complaints; ICPC-2, International Classification of Primary Care, version 2; VAS, visual analog scale; EQ-5D, EuroQol-5 dimensions quality of life scale.

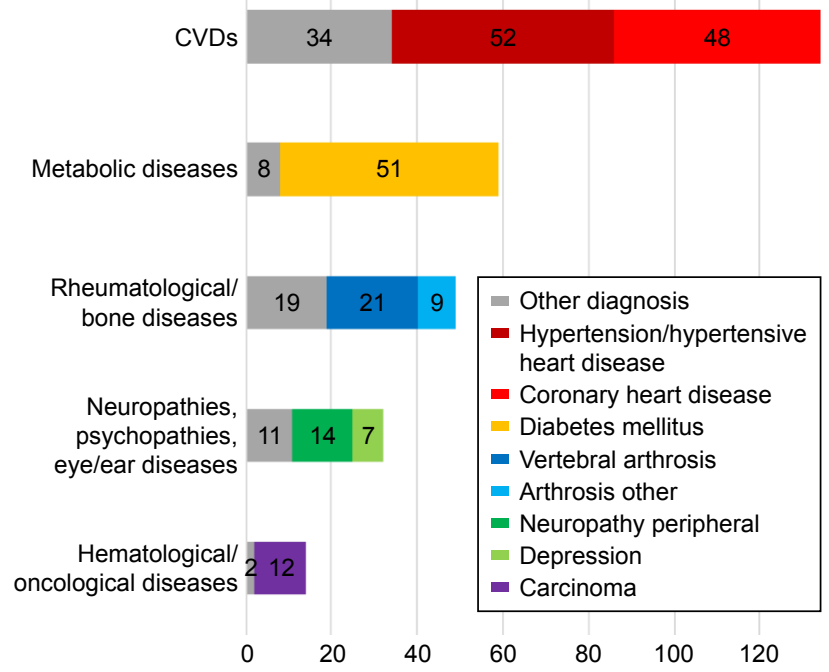

Figure 2 Diagnoses on chapter and component level defined according to an adapted version of the classification system by van den Bussche et $\mathrm{al}^{16}$ ranked by GPs as the most relevant one for the patient $(n=334)$.

Note: Further diagnoses are not shown in the figure due to low frequencies: various $(n=13)$, digestive $(n=12)$, respiratory $(n=12)$, venous $(n=7)$ and urological diseases $(n=2)$.

Abbreviations: CVDs, cardiovascular diseases; GP, general practitioner.

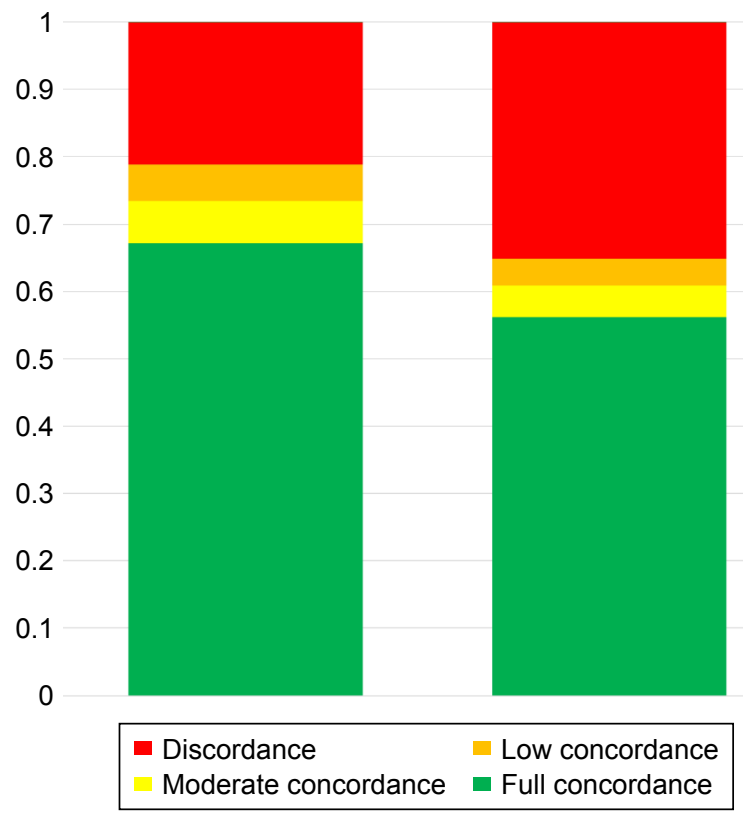

Figure 3 Patient-GP concordance of CCs according to chapter and component levels of the ICPC-2 codes.

Notes: Full concordance: patient's CC code ranked first on GP's list; moderate concordance: ranked second; low concordance: ranked third or fourth. Discordance: patient's CC code not listed on GP's list (intervention group, $n=\mid 28$ ).

Abbreviations: CC, chief complaint; GP, general practitioner; ICPC-2, International Classification of Primary Care, version 2.

while on the component level, this effect was not significant any more. Concordance between patients' ratings of CCs and GPs' ranked diagnoses on the chapter level decreased with patient's age (OR 0.96, CI 0.94-0.99, $P=0.01$ ) and increased with the number of drugs (OR 1.14, CI 1.04-1.26, $P=0.01$ ). No other association was found between covariates and the concordance with regard to CCs and between CCs and diagnoses (Table 3). In the "concordance" group, the most frequent CC was pain $(49 / 101 ; 48.5 \%)$, followed by shortness of breath $(8 / 101 ; 7.9 \%)$, weakness/tiredness $(7 / 101 ; 6.9 \%)$ and dizziness $(5 / 101 ; 4.9 \%)$. In the "discordance" group, the most frequent $\mathrm{CC}$ was pain $(8 / 27 ; 29.6 \%)$, followed by weakness/tiredness $(3 / 27 ; 11.1 \%)$ and a variety of CCs with a low frequency (ie, $1 / 27$ each; 3.7\%); shortness of breath was never listed. Between groups, only the frequency of pain ( $P=0.0005$ ) and shortness of breath (as given earlier) were significantly different.

\section{Discussion}

The concordance between patients' and GPs' ratings of CCs was high: GPs ranked the health problem, which patients declared as their most important problem, in $67 \%$ in first rank as well. Considering the complete four-part list of patients' problems, the perception of patients' CCs by GPs increased 
to $79 \%$. Thus, a majority of GPs perceive the subjective problems of their multimorbid patients correctly. This awareness is an important prerequisite for setting priorities and treatment goals together with the patient. Very recently, a Swiss study by Déruat-Luyet et a $1^{18}$ among 888 multimorbid patients and their GPs reported a concordance of $86 \%$ with regard to health conditions "bothering the patient" (A Déruat-Luyet, University of Lausanne, personal communication, July, 2017). This is in line with our findings that, as soon as GPs focus more on subjective conditions than strictly on medical terms, their perception of patients' complaints is increasing substantially. Moreover, a high awareness of patients' subjective needs may help GPs to overcome patients' unvoiced agenda, which is a major factor affecting health outcomes. ${ }^{19}$

The concordance (or discordance) between patients' ratings of CCs and GPs' ranked diagnoses was substantially lower. This finding is also reflected by differences in the categories of the most important diagnoses and CCs: While the most important diagnoses were cardiovascular, metabolic and degenerative (joint) diseases, CCs were mainly (musculoskeletal) pain and general problems, such as fatigue and breathing problems.

Zulman et $\mathrm{al}^{9}$ noted in a sample of hypertensive diabetes patients that providers ranked the patient's most important concern in their lists of three main health conditions in $72 \%$, but subjective conditions such as pain, discomfort, anxiety or breathing problems often were not perceived by GPs (ie, not appearing on GP's list). The most frequently mentioned concern of GPs in this study was hypertension. This suggests that concerns of prognosis and complications are important drivers of GPs' misperception of patients' subjective conditions, as also stated by Voigt et al. ${ }^{11}$ Junius-Walker et al ${ }^{12}$ found a poor agreement between patients and GPs on the priority of individual health problems (Cohen's $\kappa 0.11$ ), due to different concepts: the strongest predictor of a problem's relevance for patients was the emotional experience, while for GPs it was an unfavorable prognosis. ${ }^{12}$ On the other hand, Cheraghi-Sohi et $\mathrm{al}^{20}$ reported that also patients care about prognosis: the main drivers of prioritization in the perspective of patients were 1) functional health (namely, the ability "to do the things one wants or needs to do") and 2) the risk of future serious complications, loss of independence and/or death. ${ }^{20}$ The focus on diagnostic and treatment procedures as well as on prognosis might be a consequence of physicians' professional education, which traditionally favors knowledge and skills in these dimensions rather than in empathy. Fortunately, there are efforts for a change toward a more patient-centered approach in undergraduate medical education. ${ }^{21}$
Pain was by far the most frequent $\mathrm{CC}$ in our study sample, the majority of which was back pain and pain in the lower extremities, followed by weakness/tiredness and shortness of breath. Ratings of pain CC severity were not significantly different from ratings of non-pain CCs (data not shown). To our knowledge, our study is the first study investigating the frequency of CCs among multimorbid patients, strictly in patients' perspective. Thus, no direct comparison to other prevalence studies is possible, as they focus more on single complaints (as pain), health conditions, diagnoses or disease patterns, ${ }^{18,22-25}$ and not on various subjective perception of illness or suffering. The best proxy for comparison may be "reason for encounter" (RFE), assuming that CCs frequently motivate patients to see their GP. In a Danish population, general problems have been identified as the most common RFE on the ICPC chapter level, followed by musculoskeletal and respiratory problems. ${ }^{23}$ Similarly to our findings, Tandjung et $\mathrm{al}^{24}$ reported musculoskeletal problems as the most common RFE, followed by cardiovascular and respiratory problems in a Swiss primary care population. Concerning pain, there are consistent data showing that a majority of multimorbid elderly patients suffer from pain, mostly low back pain. ${ }^{25-27}$ Consequently, better addressing chronic pain in a multifaceted way should be emphasized in pre- and postgraduate training of physicians for improving multimorbid patients' quality of life, patient satisfaction, adherence to treatment and disease outcomes..$^{5-7}$

Frequency of diseases among multimorbid patients varies according to the coding system and needs careful harmonization of the definition of each chronic medical condition to allow comparison between studies. ${ }^{22}$ In our study sample, CVD - mostly hypertension and coronary heart disease was by far the most frequent diagnosis perceived as the most relevant one by the GPs, followed by metabolic and rheumatological/bone diseases (Figure 1). Typically, these conditions are clustering among multimorbid patients. ${ }^{28}$ Three huge German and one Swiss cohort study with elderly multimorbid patients confirmed this ranking, ${ }^{16,18,29}$ with one exception. Lipid disorders were listed as the most relevant diagnoses in only one of 334 patients $(0.3 \%)$ of our sample, compared to a prevalence of $40 \%-60 \%$ in the abovementioned cohorts. This finding reflects that by prioritization GPs did not rate lipid disorders as a relevant health problem for their multimorbid patients competing with other diagnoses. The comparison between concordance and discordance groups concerning CC showed a higher frequency of pain and shortness of breath in the concordance group. These normally are complaints with a high burden of illness for 
the patient and therefore the perception among GPs may be higher than for other complaints. On the other hand, no CC in the discordance group was significantly more frequent compared to the concordance group. With other words, there is no CC GPs routinely miss in their perception.

In our regression analysis, we found that a higher rating of the CC's severity by the patient had a positive impact on the patient-provider concordance of CCs. This seems plausible, as patients with a higher burden of disease may communicate about their suffering to their GPs more intensively. Lesho et $\mathrm{al}^{30}$ reported a significant discordance in different domains of subjective suffering between physicians' perception and patients' descriptions, most markedly when patients' level of suffering was mild - thus similar to our results. ${ }^{30}$ Concerning the concordance of $\mathrm{CCs}$ and ranked diagnoses, we found that age was inversely, and the number of drugs positively, associated. This may indicate that in younger age, patients and GPs similarly emphasize prognosis and are seeking agreement on it. A high number of drugs were associated with a higher concordance between CCs and diagnoses, but not with a higher patient-provider concordance of CCs. The latter fact may potentially be explained by a power problem (smaller subgroup). This finding is somehow difficult to interpret, as no data in the literature are available. The higher patient-provider concordance in patients with a higher number of drugs might reflect the need of GPs to prioritize in such situations. Hansen et $\mathrm{al}^{29}$ reported on sex, age, education, income, disease count, depression, EuroQoL VAS and nursing care dependency as factors associated with positive agreement between patients and their GPs concerning chronic diseases. ${ }^{29}$ The same research group showed in a qualitative study that the agreement between patients and GPs on relevance of illness depended on several major topics, eg, communication and cooperation between health care professionals, the communication between GP and patients and patients' disease knowledge. ${ }^{31}$

\section{Strengths and limitations}

To our knowledge, our study is the first investigating the frequency of CCs among multimorbid patients, directly considering patients' perspective. In addition, we asked GPs to prioritize patients' diagnoses, which gives a more accurate estimate of the relevance of diagnoses in multimorbid patients in the perspective of the GPs.

Another strength of our study is that we asked patients and GPs exactly the same question with regard to patients' CC. In doing so, we were able to compare the perspectives of patients and GPs one-to-one.
A limitation is the possible bias of GPs in the recruitment procedure, ie, GPs more interested in patient centeredness may have been more prone to participate in a study on multimorbid patients. Moreover, asking GPs for patients' complaints by questionnaire may have increased their attention for this matter, leading to a bias. Another limitation is the fact that some few complaints (eg, shortness of breath) are ambiguous concerning their assignment to a specific diagnoses or organ system. Thus, misclassification cannot be excluded entirely, although we tried to minimize this confounder by in-depth analysis of these single ambiguous cases.

\section{Conclusion}

A majority of GPs perceive the CC of the multimorbid patient correctly as the health condition from which the patient is suffering the most. There is no specific complaint GPs would not perceive routinely. On the other hand, there is room for improvement: $21 \%$ of GPs do not list the CC of the patient at all in a four-part list. To improve awareness of the multimorbid patient's most demanding illness, we recommend to ask for the patient's CC in every encounter and to provide room (including time) for dealing with the answer. How to manage pain, as the most frequent CC in our study, should be considered in a multifaceted approach. Increasing GPs' perception of subjective suffering of patients may translate into an improved coping with multimorbidity and into a higher quality of care for multimorbid patients.

\section{Acknowledgments}

The Gottfried and Julia Bangerter-Rhyner Foundation granted funding for this study. The author Stefan Zechmann has equal first authorship.

\section{Disclosure}

The authors report no conflicts of interest in this work.

\section{References}

1. May CR, Eton DT, Boehmer K, et al. Rethinking the patient: using Burden of Treatment Theory to understand the changing dynamics of illness. BMC Health Serv Res. 2014;14:281.

2. Man MS, Chaplin K, Mann C, et al. Improving the management of multimorbidity in general practice: protocol of a cluster randomised controlled trial (The 3D study). BMJ Open. 2016;6(4):e011261.

3. Wallace E, Salisbury C, Guthrie B, Lewis C, Fahey T, Smith SM. Managing patients with multimorbidity in primary care. BMJ. 2015; 350:h176.

4. Mead N, Bower P. Patient-centredness: a conceptual framework and review of the empirical literature. Soc Sci Med. 2000;51(7):1087-1110.

5. Francis V, Korsch BM, Morris MJ. Gaps in doctor-patient communication. Patients' response to medical advice. N Engl J Med. 1969;280(10) $535-540$. 
6. Griffith S. A review of the factors associated with patient compliance and the taking of prescribed medicines. Br J Gen Pract. 1990; 40(332):114-116.

7. Vermeire E, Hearnshaw H, Van Royen P, Denekens J. Patient adherence to treatment: three decades of research. A comprehensive review. J Clin Pharm Ther. 2001;26(5):331-342.

8. Lawn S. Compliance, concordance, and patient-centered care. Patient Prefer Adherence. 2011;5:89-90.

9. Zulman DM, Kerr EA, Hofer TP, Heisler M, Zikmund-Fisher BJ. Patient-provider concordance in the prioritization of health conditions among hypertensive diabetes patients. J Gen Intern Med. 2010; 25(5):408-414.

10. Gabbay M, Shiels C, Bower P, Sibbald B, King M, Ward E. Patient-practitioner agreement: does it matter? Psychol Med. 2003; 33(2):241-251.

11. Voigt I, Wrede J, Diederichs-Egidi H, Dierks ML, Junius-Walker U. Priority setting in general practice: health priorities of older patients differ from treatment priorities of their physicians. Croat Med J. 2010; 51(6):483-492.

12. Junius-Walker U, Stolberg D, Steinke P, Theile G, Hummers-Pradier E, Dierks ML. Health and treatment priorities of older patients and their general practitioners: a cross-sectional study. Qual Prim Care. 2011; 19(2):67-76.

13. Hasler S, Senn O, Rosemann T, Neuner-Jehle S. Effect of a patientcentered drug review on polypharmacy in primary care patients: study protocol for a cluster-randomized controlled trial. Trials. 2015; $16: 380$.

14. SecuTrial ${ }^{\circledR}$ [homepage on the Internet]. Available from: http://www. secutrial.com/. Accessed February 06, 2017.

15. O'Halloran J, Miller GC, Britt H. Defining chronic conditions for primary care with ICPC-2. Fam Pract. 2004;21(4):381-386.

16. van den Bussche H, Koller D, Kolonko T, et al. Which chronic diseases and disease combinations are specific to multimorbidity in the elderly? Results of a claims data based cross-sectional study in Germany. BMC Public Health. 2011;11:101.

17. R Core Team [homepage on the Internet]. $R$ : A Language and Environment for Statistical Computing. Austria: R Foundation for Statistical Computing V; 2013. Available from: http://www.R-project.org/. Accessed July 21, 2017.

18. Déruat-Luyet A, N'Goran AA, Herzig L. Multimorbidity in primary care from GPs' and patients' perspectives: a national database. Rev Med Suisse. 2016;12(518):922, 924-927.
19. Barry CA, Bradley CP, Britten N, Stevenson FA, Barber N. Patients' unvoiced agendas in general practice consultations: qualitative study. BMJ. 2000;320(7244):1246-1250.

20. Cheraghi-Sohi S, Morden A, Bower P, et al. Exploring patient priorities among long-term conditions in multimorbidity: a qualitative secondary analysis. SAGE Open Med. 2013;1:2050312113503955.

21. SCLO [homepage on the Internet]. Swiss Catalogue of Learning Objectives for Undergraduate Medical Training. 2008. Available from: http:// sclo.smifk.ch. Accessed on February 06, 2017.

22. Zellweger U, Bopp M, Holzer BM, Djalali S, Kaplan V. Prevalence of chronic medical conditions in Switzerland: exploring estimates validity by comparing complementary data sources. BMC Public Health. 2014; $14: 1157$.

23. Moth G, Olesen F, Vedsted P. Reasons for encounter and disease patterns in Danish primary care: changes over 16 years. Scand J Prim Health Care. 2012;30(2):70-75.

24. Tandjung R, Hanhart A, Bartschi F, et al. Referral rates in Swiss primary care with a special emphasis on reasons for encounter. Swiss Med Wkly. 2015;145:w14244.

25. Scherer M, Hansen H, Gensichen J, et al. Association between multimorbidity patterns and chronic pain in elderly primary care patients: a cross-sectional observational study. BMC Fam Pract. 2016;17:68.

26. Butchart A, Kerr EA, Heisler M, Piette JD, Krein SL. Experience and management of chronic pain among patients with other complex chronic conditions. Clin J Pain. 2009;25(4):293-298.

27. Slattery BW, O'Connor L, Haugh S, et al. Prevalence, impact and cost of multimorbidity in a cohort of people with chronic pain in Ireland: a study protocol. BMJ Open. 2017;7(1):e012131.

28. Laux G, Kuehlein T, Rosemann T, Szecsenyi J. Co- and multimorbidity patterns in primary care based on episodes of care: results from the German CONTENT project. BMC Health Serv Res. 2008;8:14.

29. Hansen H, Schafer I, Schon G, et al. Agreement between self-reported and general practitioner-reported chronic conditions among multimorbid patients in primary care - results of the MultiCare cohort study. BMC Fam Pract. 2014;15:39.

30. Lesho E, Foster L, Wang Z, et al. The accuracy of physicians' perceptions of patients' suffering: findings from two teaching hospitals. Acad Med. 2009;84(5):636-642.

31. Hansen H, Pohontsch N, van den Bussche H, Scherer M, Schafer I. Reasons for disagreement regarding illnesses between older patients with multimorbidity and their GPs - a qualitative study. BMC Fam Pract. $2015 ; 16: 68$
Patient Preference and Adherence

\section{Publish your work in this journal}

Patient Preference and Adherence is an international, peer-reviewed, open access journal that focuses on the growing importance of patient preference and adherence throughout the therapeutic continuum. Patient satisfaction, acceptability, quality of life, compliance, persistence and their role in developing new therapeutic modalities and compounds to optimize

\section{Dovepress}

clinical outcomes for existing disease states are major areas of interest for the journal. This journal has been accepted for indexing on PubMed Central. The manuscript management system is completely online and includes a very quick and fair peer-review system, which is all easy to use. Visit http://www. dovepress.com/testimonials.php to read real quotes from published authors. 